\title{
Local Group timing in Milgromian dynamics
}

\section{A past Milky Way-Andromeda encounter at $z>0.8$}

\author{
H. Zhao ${ }^{1}$, B. Famaey ${ }^{2}$, F. Lüghausen ${ }^{3}$, and P. Kroupa ${ }^{3}$ \\ 1 SUPA, School of Physics and Astronomy, University of St Andrews, UK \\ e-mail: hz4@st-andrews.ac.uk \\ 2 Observatoire Astronomique, Université de Strasbourg, CNRS UMR 7550, Strasbourg, France \\ e-mail: benoit.famaey@astro.unistra.fr \\ 3 AIfA, Universität Bonn, 53121 Bonn, Germany \\ Received 12 May 2013 / Accepted 5 July 2013
}

\begin{abstract}
Context. Local Group (LG) timing is one of the first historical probes of the so-called missing mass problem. Whilst modern cosmological probes indicate that pure baryonic dynamics is not sufficient on the largest scales, nearby galaxies and small galaxy groups persistently obey Milgrom's MOND law, which implies that dynamics at small scales is possibly entirely predicted by the baryons. Aims. Here, we investigate LG timing in this context of Milgromian dynamics.

Methods. We used the latest measured proper motions and radial velocities for Andromeda and the Magellanic clouds, and we integrated their orbits backwards by making use of the Milgromian two-body equation of motion.

Results. With the currently measured proper motions and radial velocity of M31, we find that MOND would imply that the Milky Way (MW) and M31 first moved apart via Hubble expansion after birth, but then necessarily were attracted again by the Milgromian gravitational attraction, and had a past fly-by encounter before coming to their present positions. This encounter would most probably have happened 7 to $11 \mathrm{Gyr}$ ago $(0.8<z<3)$. The absence of a dark matter halo and its associated dynamical friction is necessary for such a close encounter not to have triggered a merger. Observational arguments which could exclude or favour such a past encounter would thus be very important in order to falsify or vindicate Milgromian dynamics on the scale of the LG. Interestingly, the closest approach of the encounter is small enough $(<55 \mathrm{kpc})$ to have had severe consequences on the disc dynamics, including perhaps thick disc formation, and on the satellite systems of both galaxies. The ages of the satellite galaxies and of the young halo globular clusters, all of which form the vast polar structure around the MW, are consistent with these objects having been born during this encounter.
\end{abstract}

Key words. galaxies: groups: individual: Local Group - Galaxy: evolution - gravitation

\section{Introduction}

Local Group (LG) timing (e.g. Lynden-Bell 1981; Peebles 1989) is one of the historical probes of the missing mass problem. At the end of the 1950s, Kahn \& Woltjer (1959) noted that the Milky Way (MW) and Andromeda (M31) galaxies were approaching each other, thus overcoming cosmic expansion. By assuming that they were initially formed close together they concluded that the MW-M31 system had to be at least 20 times more massive than the stellar mass to actually overcome cosmic expansion and reach its current velocity and position. In its simplest version, the LG consists of the MW and M31 as two isolated point masses that moved apart because of Hubble expansion, then slowed down and moved towards each other again, which could not possibly have happened in Newtonian gravity without dark matter (DM).

Since then, numerous other pieces of evidence, culminating with the latest data release of the Planck mission (Planck Collaboration 2013), have accumulated to indicate the apparent need for missing non-baryonic fields (behaving as a dissipationless dust fluid) on the largest scales of the Universe. However, this does not necessarily and unequivocally imply the existence of stable neutral DM particles at galactic scales. The observed phenomenology in a wide range of near-field galaxy data (e.g. Famaey \& McGaugh 2012) indicates that the gravitational field can be entirely predicted by the baryons, independently of the history and environment of each galaxy. This is at odds with the a priori predictions from collisionless DM particles since it would then imply a large amount of fine-tuning in the baryonsfeedback for a large sample of independent systems. This adds up to the numerous other small-scale problems reviewed in e.g. Kroupa et al. (2010).

An alternative is to consider that small-scale dynamics is fundamentally governed by the empirical law of Milgrom (1983), also known as MOdified Newtonian Dynamics (MOND), which summarizes the above phenomenology by stating that for gravitational accelerations below $a_{0} \simeq 10^{-10} \mathrm{~m} \mathrm{~s}^{-2}$, the effective gravitational attraction $g$ approaches $\left(g_{\mathrm{N}} a_{0}\right)^{1 / 2}$ where $g_{\mathrm{N}} \propto 1 / r^{2}$ is the usual Newtonian gravitational acceleration calculated from the observed distribution of baryonic matter. This might be reconciled with cosmological data through covariant theories involving e.g. massive vector fields (e.g. Zhao \& Li 2010), bimetric theories with twin matter fields (e.g. Milgrom 2009), the presence of a dipolar dark fluid (e.g. Blanchet \& Le Tiec 2009), etc. These fields are fundamentally different from cold DM particles on galaxy scales; for instance, they would not induce dynamical friction in galaxies between the stars and DM particles, contrary to what happens in the standard context, and in the weak-field limit all these theories do lead to the MOND phenomenology. To reconcile these theories with galaxy clusters, one might resort to the non-trivial effect of these new fields on large scales (e.g. Dai et al. 2008), or to additional particles that would not cluster on 
galaxy scales (e.g. Angus et al. 2007). On the other hand, on the scale of galaxy groups, the data seem to be in agreement with the MOND phenomenology without any addition (Milgrom 2002). More generally, weak lensing on the scales of hundreds of kpc indicates that the effective gravity falls off as $1 / r$ for luminosities comparable to those of the LG (Brimioulle et al. 2013; Milgrom 2013). We thus investigate here what this phenomenology would imply for the history of the LG.

Motivated by the recent Hubble Space Telescope (HST) measurement of the proper motion of M31 (Sohn et al. 2012) that has considerably reduced the error bars, we investigated the timing argument in the MOND context. Precise measurements of the proper motion of the Magellanic clouds were also obtained (Kallivayalil et al. 2013). These HST measurements have led to a few recent studies (i) that estimated the dynamical mass of the LG in the standard context (van der Marel et al. 2012a) and showed that the timing argument led to an increase in dynamical mass of about $10 \%$ with respect to other estimates; (ii) that studied the future encounter of the MW and M31 (van der Marel et al. 2012b); or (iii) that studied the variety of solutions allowed for the history and dynamics of the LG (Peebles \& Tully 2013).

In the context of MOND the situation is slightly different as there is not as much freedom on the baryonic masses of galaxies as there is on their putative DM masses in the standard context. Once we know the baryonic masses, distances, and velocities of galaxies, as well as the external field from the large-scale structure, a unique history can be traced backwards. Making use of the Milgromian two-body equation of motion, we thus investigate what these recent measurements would imply in the context of MOND. In Sect. 2, we derive the equation of motion in the context of Hubble expansion; we then integrate backwards the orbits of some LG galaxies in Sect. 3, and discuss the results in Sect. 4.

\section{Two-body equation of motion in a cosmological context}

Even in their classical non-covariant form, MOND theories (Bekenstein \& Milgrom 1984; Milgrom 2010) are inherently non-linear and require us to solve complicated modified Poisson field equations (e.g. Tiret \& Combes 2007; Lüghausen et al. 2013). But in a simple two-body configuration of two point-like masses $m_{1}$ and $m_{2}$ separated by a distance $r_{12}$, they boil down to a two-body force of the form (see, e.g. Milgrom 1994; Zhao \& Famaey 2010; Zhao et al. 2010)

$F_{12}=\frac{G m_{1} m_{2}}{r_{12}^{2}}\left[1+y^{-1 / 2}\right], y \equiv\left[\frac{\sqrt{G\left(m_{1}+m_{2}\right) a_{0}}}{r_{12} Q a_{0}}\right]^{2}$,

where $Q \equiv \frac{2\left(1-q_{1}^{3 / 2}-q_{2}^{3 / 2}\right)}{3 q_{1} q_{2}}$ and $q_{1} \equiv 1-q_{2} \equiv \frac{m_{1}}{m_{1}+m_{2}}$. This holds completely rigorously if the interpolating function is of Bekenstein's form (see, e.g. Zhao \& Famaey 2010; Famaey \& McGaugh 2012); however, for an orbit mostly in the deep-MOND $\left(g<a_{0}\right)$ regime, the exact form of the interpolating function is of little importance.

Considering the expansion of the background universe $a(t)$ the equation of motion for the relative separation (Zhao et al. 2010) is then given by

$\frac{\mathrm{d}^{2}}{\mathrm{~d} t^{2}} \boldsymbol{r}_{12}=K \boldsymbol{r}_{12}-\frac{m_{1}+m_{2}}{m_{1}}\left[\frac{\boldsymbol{F}_{12}}{m_{2}}\right], K \equiv \frac{\mathrm{d}^{2} a}{a \mathrm{~d} t^{2}}$,

where $F_{12}$ is their mutual force, and their distance in proper coordinates $r_{12}=\left|\boldsymbol{r}_{1}-\boldsymbol{r}_{2}\right|=\left|\boldsymbol{x}_{1}-\boldsymbol{x}_{2}\right| a(t)$. We note that there

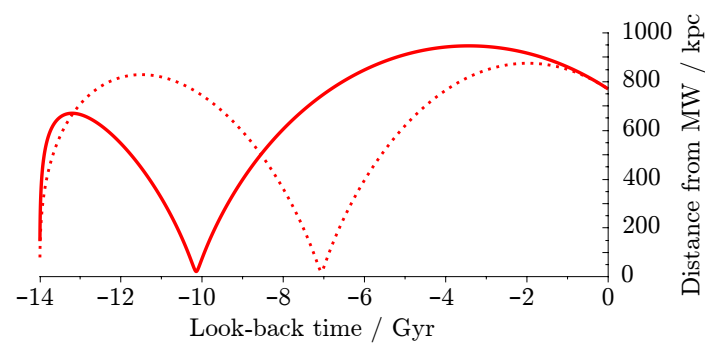

Fig. 1. Evolution of the relative distance between the MW and M31 as a function of time for the nominal parameters in the first line of Table 1. Dotted line: negligible external field. Solid line: external field from the large-scale structure adopted to be $3 \%$ of $a_{0}$ (Wu et al. 2008).

would be a frictional term $(\mathrm{d} a / \mathrm{d} t)\left(\mathrm{d} \boldsymbol{x}_{i} / \mathrm{d} t\right)$ for the equation of motion in comoving coordinates. This term does not exist when the equation is written for the proper coordinates. The remaining cosmological term is $K=n(n-1) t^{-2}$ if the cosmic expansion factor is approximated as a power law $a(t)=\left(t / t_{0}\right)^{n}$. Empirically adopting an expansion of the Universe from standard cosmology, $\mathrm{d} a /(a \mathrm{~d} t)=(1 / 14 \mathrm{Gyr}) \sqrt{0.667+0.333 a^{-3}}$, we find the approximation

$K=\frac{\mathrm{d}^{2} a}{a \mathrm{~d} t^{2}}=\frac{2}{3(14 \mathrm{Gyr})^{2}}-\frac{2}{9 t^{2}}$,

such that the universe was decelerating at early times when $a(t) \propto t^{2 / 3}$, and is exponentially accelerating at late times. Using Eqs. (1)-(3), orbits can then be integrated backwards in $3 \mathrm{D}$ as per Zhao et al. (2010). In Sect. 3.2 we also consider the effect of the external field acting on the LG, estimated from arguments based on the local galactic escape speed from the solar neighbourhood and estimations of the actual gravity of the large-scale structure (Famaey et al. 2007; Wu et al. 2008).

\section{Results}

\subsection{M31-Milky Way orbit}

We applied Eqs. (1)-(3) to the M31-MW system. Contrary to the Newtonian DM case, there is not much freedom on the masses $m_{1}$ and $m_{2}$ of the galaxies that we parametrized in MOND through their asymptotically flat circular velocities $V_{\infty i}=\left(G m_{i} a_{0}\right)^{1 / 4}$ adopted from Wu et al. (2008) and Carignan et al. (2006), see Table 1. These parameters control the period of the orbit. The current distance of M31 has been adopted to be $770 \mathrm{kpc}$ (Karachentsev et al. 2004), which also influences the period.

The other fundamental parameters are the current radial and tangential velocity of M31 with respect to the MW. The first is known to be $V_{r}=109.3 \pm 4.4 \mathrm{~km} \mathrm{~s}^{-1}$, which we adopt here neglecting the small error, while the second is $V_{T}=17 \pm 17 \mathrm{~km} \mathrm{~s}^{-1}$ at 1 sigma (Sohn et al. 2012; van der Marel et al. 2012a) that we vary as stated in Table 1 . This parameter controls the angular momentum, hence the closest approach distance.

First, a total of 12 models were considered (listed in Table 1), varying the parameters within realistic error bars, and the very robust conclusion is that MOND unavoidably implies that the MW and M31 had a past encounter. The evolution of the MW-M31 distance with time for the nominal parameters (1st line of Table 1) is illustrated as a dotted line in Fig. 1 (with an encounter $\sim 7$ Gyr ago).

In principle, this past close encounter is also unavoidable for untruncated isothermal halos up to scales of several hundreds of kpc, but it would actually be impossible in the Newtonian dark 
Table 1. Parameters adopted for 12 models of the M31-MW system.

\begin{tabular}{llcccc}
\hline \hline$V_{\infty}^{\mathrm{MW}}\left(m_{\mathrm{b}}\right)$ & $V_{\infty}^{\mathrm{M} 31}\left(m_{\mathrm{b}}\right)$ & $V_{T}^{\mathrm{M} 31}$ & $b$ & $T_{\text {enc }}($ no EF $)$ & $T_{\text {enc }}\left(\mathrm{EF}=0.03 a_{0}\right)$ \\
\hline $\mathbf{1 8 0}(\mathbf{0 . 7})$ & $\mathbf{2 2 5}(\mathbf{1 . 6})$ & $\mathbf{1 7 - 3 4}$ & $\mathbf{2 2 - 4 8}$ & $-\mathbf{7 .}$ & $\mathbf{- 1 0 . 2}$ \\
$180(0.7)$ & $215(1.4)$ & $17-34$ & $19-56$ & -7.3 & -11.2 \\
$180(0.7)$ & $205(1.1)$ & $17-34$ & $22-55$ & -7.7 & -12.3 \\
$190(0.85)$ & $250(2.5)$ & $17-34$ & $19-47$ & -6. & -7.9 \\
$170(0.55)$ & $225(1.6)$ & $17-34$ & $26-54$ & -7.2 & -11.0 \\
$180(0.7)$ & $225(1.6)$ & $0-10$ & $0-13$ & -7. & -10.2 \\
\hline
\end{tabular}

Notes. Columns 1, 2: asymptotic flat circular velocities of the MW and M31 in $\mathrm{km} \mathrm{s}^{-1}$, related to the baryonic masses through $m_{b}=V_{\infty}^{4} /\left(G a_{0}\right)$, given after in units of $10^{11} M_{\odot}$; Cols. 3, 4: adopted range for the current tangential velocity of M31 with respect to us, in $\mathrm{km} \mathrm{s}^{-1}$, and the corresponding impact parameter $b$ in kpc; Cols. 5, 6: look-back time of the encounter $T_{\text {enc }}$ in Gyr, assuming the LG is in a negligible external field and in a non-negligible external field of $3 \%$ of $a_{0}$, respectively.

halo context. Indeed, a close encounter (with a closest approach between 0 and $56 \mathrm{kpc}$ ) would inevitably lead to the merging of the two extended colliding halos as a result of dynamical friction between their massive particles. In the context of MOND, however, the dynamical friction, while slightly enhanced within the stellar discs of the galaxies, would be negligible outside of it. As shown by the simulations of Tiret \& Combes (2008), mergers would last longer in MOND and imply multiple passages of the merging galaxies. A closest approach from 20 to $55 \mathrm{kpc}$ would certainly have dynamical influences on the two colliding galaxies but would not trigger a merger in MOND, keeping in mind that stellar discs would be $60 \%$ to $70 \%$ of their current size at that epoch (e.g. van Dokkum et al. 2013). We note that low tangential velocities $\left(\sim 10 \mathrm{~km} \mathrm{~s}^{-1}\right.$ and below) in Table 1 , however, lead to close encounters which would probably trigger a merger in MOND too, through stellar dynamical friction; MOND thus predicts that the tangential velocity of M31 cannot be much smaller than the measured $17 \mathrm{~km} \mathrm{~s}^{-1}$.

\subsection{External field effect}

In Milgromian dynamics, an important aspect is that the internal dynamics of a system is affected by the external gravitational field in which it is embedded. In the case of the MW-M31 pair, the external gravitational field from the large-scale structure does not dominate over the internal gravity of the system along most of the orbit as long as it remains below $\sim 2 \%$ of $a_{0}$. From arguments based on the local galactic escape speed from the solar neighbourhood and estimations of the actual gravity of the largescale structure, the external field acting on the LG is between $1 \%$ and $3 \%$ of $a_{0}$ (Famaey et al. 2007; Wu et al. 2008). We thus recomputed the orbits for the latter value of the external field in such a way that when $g<0.03 a_{0}$ the gravity becomes essentially Newtonian again but with a renormalized $\tilde{G} \approx G / 0.03$. The effect is similar to truncating a Newtonian isothermal halo (Wu et al. 2008).

To ensure a general and smooth interpolation of the two-body force between the strong, weak, and external-field dominated regimes, we considered the following empirical equation:

$F_{12} \approx \frac{\tilde{G} m_{1} m_{2}}{r_{12}^{2}}, \tilde{G} \equiv G\left[1+\left(y+\frac{g_{\mathrm{ext}}^{2}}{a_{0}^{2}}\right)^{-\alpha}\right]^{\frac{1}{2 \alpha}}$,

where $y$ is defined as in Eq. (1). Here the parameter $\alpha$ plays the role of the interpolating function; setting $\alpha=1 / 2$ and $g_{\text {ext }}=0$ gives back precisely Eq. (1). We checked that by adopting $\alpha=1$ and $g_{\mathrm{ext}}=0$, the closest approach and encounter epoch of M31 were almost unchanged, confirming that the exact form of the interpolating function is of little importance when most of the orbit is in the deep-MOND regime. We then considered a solution for $\alpha=1$ and $g_{\mathrm{ext}}=0.03 a_{0}$. For this solution, gravity thus becomes weaker in the outskirts of the orbit than without external field, and this makes the period longer. The encounter is thus pushed back to earlier times, as listed in the last column of Table 1. Interestingly, the closest approach is almost unchanged (by less than $2 \mathrm{kpc}$ ) compared to the no-external field case. Also, the corresponding launch speeds calculated by integrating backwards are smaller for this non-negligible external field solution, meaning that the first encounter happens sooner after the Big Bang than in the case without external field.

For the external-field solution with the nominal values of the parameters (1st line of Table 1), the encounter took place 10.2 Gyr ago. It is compared (solid line) to the noexternal field solution for the same parameters (dotted line) in Fig. 1. This is particularly interesting since this timing of the encounter roughly corresponds to the age of most dwarf satellites of the LG, bringing with it the possibility that these were created during the event as tidal dwarf galaxies (see e.g. Kroupa et al. 2010; Pawlowski et al. 2012, and references therein). As an example, we investigate the orbits of the Magellanic clouds in MOND to see whether their orbits would possibly be close to pericenter at the time of the encounter.

\subsection{Magellanic clouds}

Applying the same method, in the simplifying case of a twobody configuration where the orbit of the Large Magellanic Cloud (LMC) around the MW is unperturbed by the rest of the LG, we found that the nominal proper motion (Kallivayalil et al. 2013) of the LMC (presently at a distance of $49 \mathrm{kpc}$ ) would imply a period of about 3.5 Gyr in MOND without external field, and $\sim 5$ Gyr for an external field of $0.03 a_{0}$ (and $\alpha=1$ ). In both cases, the nominal proper motions from Kallivayalil et al. (2013) neatly puts it quite close to pericenter at the time of the MW-M31 encounter. In Fig. 2, we plot the joint evolution of the distance to the MW of the LMC together with that of M31 for the solution with external field. We also show the planar shape of the orbits (lower panel of Fig. 2). While sensitive to the exact value of the proper motion, several solutions are found where the two orbits (of M31 and the LMC) would cross near the $Z$-axis, which would imply a strong orbital perturbation of the LMC and perhaps a gas shock.

The Small Magellanic Cloud (SMC), presently at a distance of $63 \mathrm{kpc}$, is considered here an independent object unperturbed by the LMC, and is then on a nearly circular orbit in MOND (Fig. 2). We note that the SMC-LMC pair is typically in a region where the external field from the MW dominates its internal dynamics, and the high relative velocity of the SMC with respect to the LMC then implies that the two are not bound in the MOND context. The Magellanic stream would then have to be due to recent ram-pressure stripping of gas from the SMC since the stream contains very few stars. A recent encounter of the LMC and SMC might have released some gas clouds from the gas-rich SMC, and these gas clouds could have been further stretched by ram-pressure with the denser clouds leading the less dense ones. In any case, at the time of the MW-M31 encounter, the three objects were thus possibly all in a region of $\sim 100 \mathrm{kpc}$ of radius, meaning that tidal interaction among these three objects (and other satellites within $100 \mathrm{kpc}$ of the MW and M31) would have been inevitable, and the Magellanic clouds and the discs of satellites (DoS) in the MW and M31 could possibly be debris objects created during this event. 


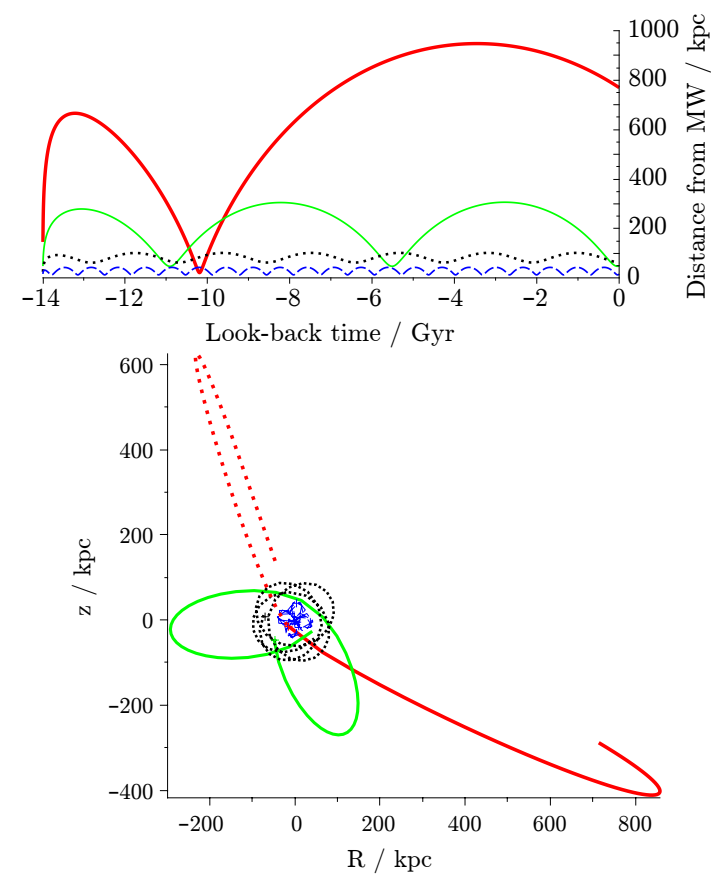

Fig. 2. Upper panel: the radial distances to M31 (thick red), LMC (thin green), and SMC (dotted black) for the past 14 Gyr for the model parameters in the first line of Table 1 and an external field of $0.03 a_{0}$. We note the MW-M31 encounter $10.2 \mathrm{Gyr}$ ago and the presence of the LMC and SMC close to pericenter at that epoch. The Sagittarius dwarf (dotted blue) orbit is also plotted for reference. Lower panel: planar shape of the orbits with respect to the MW. The thick red line is the orbit of M31. The first part of the orbit is indicated as a dotted red line, and becomes a solid red line from the time of the encounter 10.2 Gyr (marked by crosses) ago. All orbits are assumed to be on independent meridional $R Z$ planes with the horizonal axis $R= \pm \sqrt{r^{2}-Z^{2}}$ showing the offset from the MW rotation axis $Z$.

\section{Conclusion and discussion}

Here we have shown that if Milgromian dynamics is a valid effective description of gravity on the scales of hundreds of $\mathrm{kpc}$, then the latest HST measurement of the proper motion of M31 implies that it necessarily had a past close encounter with the MW 6 to $12 \mathrm{Gyr}$ ago (most probably 7 to $11 \mathrm{Gyr}$ ago; see Table 1, where encounter epochs outside of this range correspond to unrealistic baryonic masses of M31). The closest approach would be less than $\sim 55 \mathrm{kpc}$ in all cases.

Such galaxy interactions are observed to be quite common at $z \sim 1$, and Milgromian dynamics would thus imply that we might be seeing the aftermath of such an event, for instance in the peculiar orbits of the dwarf galaxies of the LG since all satellites within $100 \mathrm{kpc}$ of the MW and M31 would have been affected. In the case where the encounter happened $\sim 10 \mathrm{Gyr}$ ago, it is possible that most dwarf galaxies of the LG would have been created in the event. Such a close encounter between the MW and M31 has already been suggested by Pawlowski et al. (2012) as possibly being responsible for the creation of the vast polar structure (VPOS) of satellites around the MW. Indeed the DoS configurations suggest that the satellite galaxies in both the MW (Lynden-Bell 1982; Zhao 1998; Kroupa et al. 2010) and partly in M31 (Ibata et al. 2013) might have been formed in tidal arms (see also Hammer et al. 2013), and hence are pure concentrations of cooled baryons which could not harbour DM halos; that tidal dwarfs exhibit missing mass in Newtonian dynamics thus only makes sense in the MOND context (see also Gentile et al. 2007).
To piece together these puzzles self-consistently, it is thus particularly satisfying that such a close interaction between the MW and M31 is actually inevitable in MOND.

The LG also helps to break the often frustratingly degenerate predictions of MOND and an isothermal DM halo truncated at $\sim 100 \mathrm{kpc}$ radius. Importantly, the M31-MW encounter scenario is self-consistent only in the absence of a particle DM halo, to avoid dynamical friction that inevitably triggers a rapid merger. The lack of dynamical friction in MOND galaxy interactions has been well demonstrated by the simulations of Tiret \& Combes (2008); the key to reducing friction is to have a slightly non-head-on encounter. However, we showed that low tangential velocities (see Table 1) would lead to small impact parameters which would probably still trigger a merger through stellar dynamical friction so, in MOND, the tangential velocity of M31 cannot really be much smaller than the measured $17 \mathrm{~km} \mathrm{~s}^{-1}$. We note, however, that the first part of the orbit before the encounter and the actual original impact parameter are not necessarily well determined by the present modelling, since the conserved quantities we see today (energy and angular momentum) are only valid for point masses. Actually, there may have been a transfer of energy and angular momentum between the orbit and the internal motions during the fly-by. A small fraction of gas might also have been launched into the LG by ram pressure, which might have led to various stellar (sub)structures in the LG and in the stellar halos of both galaxies. We finally note that the origin of the thick disc of the MW is also still uncertain, and we point out that, in the MOND context, it could be the result of this same violent perturbation by M31 about 10 Gyr ago.

\section{References}

Angus, G., Shan, H., Zhao, H., \& Famaey, B. 2007, ApJ, 654, L13 Bekenstein, J., \& Milgrom, M. 1984, ApJ, 286, 7

Blanchet, L., \& Le Tiec, A. 2009, Phys. Rev. D., 80, 023524

Brimioulle, F., Seitz, S., Lerchster, M., et al. 2013, MNRAS, 432, 1046

Carignan, C., Chemin, L., Huchtmeier, W., \& Lockman, F. 2006, ApJ, 641, L109

Dai, D.-C., Matsuo, R., \& Starkman, G. 2008, Phys. Rev. D, 78, 104004

Famaey, B., \& McGaugh, S. 2012, Liv. Rev. Relativit. 15, 10

Famaey, B., Bruneton, J.-P., \& Zhao, H.S. 2007, MNRAS, 377, L79

Gentile, G., Famaey, B., Combes, F., et al. 2007, A\&A, 472, L25

Hammer, F., Yang, Y., Fouquet, S., et al. 2013, MNRAS, 431, 3543

Ibata, R., Lewis, G., Conn, A., et al. 2013, Nature, 493, 62

Kahn, F., \& Woltjer, L. 1959, ApJ, 130, 705

Kallivayalil, N., van der Marel, R. P., Besla, G., et al. 2013, ApJ, 764, 161

Karachentsev, I., Karachentseva, V., Huchtmeier, W., et al. 2004, AJ, 127, 2031

Kroupa, P., Famaey, B., de Boer K., et al. 2010, A\&A, 523, A32

Lüghausen, F., Famaey, B., Kroupa, P., et al. 2013, MNRAS, 432, 2846

Lynden-Bell, D. 1981, The Observatory, 101, 111

Lynden-Bell, D. 1982, The Observatory, 102, 202

Milgrom, M. 1983, ApJ, 270, 365

Milgrom, M. 1994, ApJ, 429, 540

Milgrom, M. 2002, ApJ, 577, L75

Milgrom, M. 2009, Phys. Rev. D., 80, 123536

Milgrom, M. 2010, MNRAS, 403, 886

Milgrom, M. 2013, Phys. Rev. Lett., 111, 041105

Pawlowski, M. S., Pflamm-Altenburg, J., \& Kroupa, P. 2012, MNRAS, 423,

1109

Peebles, J. 1989, ApJ, 344, L53

Peebles, J., \& Tully, B 2013 [arXiv: 1302 .6982]

Planck Collaboration 2013, A\&A, submitted [arXiv: 1303.5076]

Sohn, S., Anderson, J., \& van der Marel, R. P. 2012, ApJ, 753, 7

Tiret, O., \& Combes F. 2007, A\&A, 464, 517

Tiret, O., \& Combes, F. 2008, ASP Conf. Ser., 396, 259

van der Marel, R. P., Fardal, M., Besla, G., et al. 2012a, ApJ, 753, 8

van der Marel, R. P., Besla, G., Cox, T., et al. 2012b, ApJ, 753, 9

van Dokkum, P. G., Leja, J., Nelson, E., et al. 2013, ApJ, 771, L35

Wu, X., Famaey, B., Gentile, G., et al. 2008, MNRAS, 386, 2199

Zhao, H. 1998, MNRAS, 294, 139

Zhao, H., \& Famaey, B. 2010, Phys. Rev. D, 81, 087304

Zhao, H., \& Li, B. 2010, ApJ, 712, 130

Zhao, H., Li, B., \& Bienaymé, O. 2010, Phys. Rev. D, 82, 103001 